\title{
SYNDECAN-1, CD117 AND CD56 IMMUNOHISTOCHEMICAL EVALUATION OF PLASMACYTOMA
}

\author{
Sherif F. Elgayar*, Hanan G. Eid*** and Maii I. Sholqamy**
}

\begin{abstract}
Background and Objective: World Health Organization (WHO) describes two types of plasmacytomas which are solitary bone plasmacytoma (SBP) and extramedullary plasmacytoma (EMP). $17 \%$ to $33 \%$ of plasmacytomas, will progress into multiple myeloma (MM). The early diagnosis is very important as the plasmacytoma may be primary or metastatic lesion of MM. Prognosis of solitary plasmacytoma could be worse if it recurred as in case of its evolution toward systemic MM.
\end{abstract}

Methodology: 10 patients with plasmacytoma in the National Cancer Institute and Histopathological laboratory of Minia University were analyzed immunohistochemically for detection of syndecan-1 as a diagnostic marker for plasmacytoma and detection of plasmacytoma behavior through interpretation the expression of both CD117 and CD56.

Results: All cases showed strong positive immunoreaction for Syndecan-1(grade 3) according to scoring system for immunohistochemical expression. The expression of CD117 in plasmacytoma was similar to the expression of CD56, (60\%) showed grade 1, while (40\%) were grade 0 .

Conclusion: We concluded that the importance of histopathological biopsy for early diagnosis and prognostic evaluations are important in that the plasmacytoma may be a primary or metastatic lesion of MM. and Syndecan-1can be used as diagnostic marker for plasma cells, while CD56 and CD117 expressed only in neoplastic plasma cells and their expression indicate good prognosis.

KEY WORDS: plasmacytoma, syndecan 1, CD117, CD56, immunohistochemistry

\section{INTRODUCTION}

Plasmacytomas classified by World Health Organization (WHO) and is associated with the production of a monoclonal immunoglobulin or light chains. The WHO describes two types of plasmacytomas If there is absence of systemic involvement, which are solitary bone plasmacytoma (SBP) and extramedullary plasmacytoma (EMP) ${ }^{(1)}$.

The exact etiology of solitary plasmacytoma is unknown, but there are contributing factors to the

* Assistant Professor of Oral and Maxillofacial Pathology, Faculty of Oral and Dental Medicine, Minia University, Minia, Egypt.

** Lecturer of Oral and Maxillofacial Pathology, Faculty of Oral and Dental Medicine, Minia University, Minia, Egypt. 
development of the lesion as chronic stimulation, radiation overdose, viral infections and genetic interaction in the reticuloendothelial system ${ }^{(2)}$.

More than $80 \%$ of EMPs arise in the lymphatic tissue of the upper respiratory tract ${ }^{(1)}$.

EMPs are rare when compared to SBP but it has a better prognosis as the majority can be treated by local radiotherapy ${ }^{(3)}$.

Seventeen to $33 \%$ of plasmacytomas, will convert into multiple myeloma (MM) ${ }^{(1)}$. SBP may represent a primary lesion or focus of $\mathrm{MM}^{(4,5)}$.

Multiple myeloma by definition is a neoplasm of proliferating monoclonal $\mathrm{B}$ cells which are terminally differentiated (plasma cells). The conversion of mature $\mathrm{B}$ cells into malignant plasma cells is a multi-step process ${ }^{(6)}$. The time of recurrence of MM after treatment is about 2-2.5 years ${ }^{(7)}$.

The histopathological examination of SBP is very important for the definitive diagnosis ${ }^{(8)}$. As SBP are very similar histologically to ameloblastoma, keratocystic odontogenic tumor, myxoma, giant cell central lesion, metastatic tumors, vascular malformation, sarcoma and lymphoma ${ }^{(9,10)}$.

Syndecan-1 (CD138) is a multifunctional regulator of cell behavior as it regulates the growth of tumor cells, adhesion, survival and invasion(9). It also expressed on the surface of tumor cells of hematopoietic B-lineage including multiple myeloma as cell surface proteoglycan ${ }^{(8,9)}$.

Syndecan-1 was considered to be a sensitive marker for plasmacytomas, independent of cytologic differentiation; there are problems in the histologic assessment of plasmacytomas in the routine tissue sections particularly the plasmablastic type. The cells of plasmacytoma are very similar to different neoplasms ${ }^{(11)}$.

Syndecan-1 can also stains the Reed-Sternberg cells of nodular sclerosing and mixed cellularity Hodgkin's lymphoma, acquired immunodeficiency- related lymphomas, and squamous cell carcinomas, but the histologic assessment of the cutaneous plasmacytoma precludes these neoplasms in the differential diagnosis ${ }^{(12)}$.

To discriminate resemblance many markers as CD117 are used, CD117 act as a tyrosine kinase receptor protein that in humans is encoded by the KIT gene ${ }^{(8)}$, CD117 can be used to identify certain types of hematopoietic progenitors in the bone marrow as it act as cell surface marker, also different cells as hematopoietic stem cells (HSC), multipotent progenitors (MPP), and also common myeloid progenitors (CMP) express high levels of CD117 ${ }^{(9)}$.

Also The CD117 plays an important role in the proliferation of neoplastic plasma cells in multiple myeloma because it increases the number of S-phase plasma cells, and enhances the responsiveness of myeloma cell to interleukin- $6^{(10)}$

One member of a large family of cell surface glycoproteins that share structural motifs related to immunoglobulin and fibronectin type III domains is the $\operatorname{CD} 56^{(13,14)}$.

Human CD56 is encoded by single-copy gene

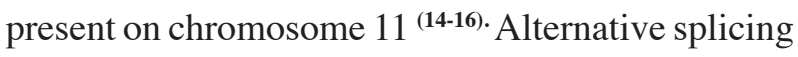
results in the expression of three major isoforms that are different in their intracellular domains and their membrane association. ${ }^{(14) .}$

CD56 was primarily characterized as a mediator of cell-cell adhesion, however recently it is also considered to be a signaling receptor that effect on cellular adhesion, proliferation, migration, apoptosis, differentiation, survival, and synaptic plasticity ${ }^{(11,17)}$.

Also CD56 is an adhesion molecule and was believed to be a marker that involved in the anchoring of plasma cells to stromal structures. Decrease in the expression of CD56 associated with malignant plasma cells and down-regulation of CD56 means increased proliferation and spreading of malignant plasma cells ${ }^{(18,19)}$. 
In this study, the syndecan-1 will be used as a diagnostic marker for plasmacytoma and detection of plasmacytoma behavior through interpretation the expression of both CD117 and CD56.

\section{MATERIAL AND METHODS}

Based on archival pathology specimens and case-note reviews a total of ten patients with plasmacytoma (eight cases were SBP and two cases were EMP) in the National Cancer Institute and Histopathological laboratory of Minia University.

Paraffin-embedded tissues 4 - $\mu$ m-thick sections were prepared, deparaffinized in xylene, and rehydrated in a graded series of alcohol. Endogenous peroxidase activity was blocked by immersing the section in $3 \%$ peroxidase, and antigens were retrieved by using a microwave (Syndecan: 10 min; CD117 and CD56:15min) with the sections in acetate buffer ( $\mathrm{pH}$ 6) as shown in fig 1 . The slides were incubated at room temperature overnight with primary antibodies (Syndecan, CD117 and CD56). After being washed with phosphate buffered saline, the sections were labeled with streptavidin-biotin for 10 minutes at room temperature. The sections were visualized using diaminobenzidine hydrochloride. Finally, the sections were counted stained with Mayer's hematoxylin.

Two experienced pathologists examined and evaluated the immune-expression of the prepared sections according to the criteria presented in table 1. ${ }^{(20)}$

TABLE (1): Scoring system for immunohistochemical expression

\begin{tabular}{|l|l|}
\hline Grade & Characterization \\
\hline \multicolumn{2}{|l|}{ Scoring system for Immunohistochemical expression } \\
\hline $\mathbf{0}$ & Absent \\
\hline $\mathbf{1}$ & Mild immunohistochemical expression \\
\hline $\mathbf{2}$ & Moderate immunohistochemical expression \\
\hline $\mathbf{3}$ & Massive immunohistochemical expression \\
\hline
\end{tabular}

\section{RESULTS}

A total number of ten cases were taken for the study, all cases showed strong positive immunoreaction for Syndecan-1(grade 3). There was cytoplasmic and membranous expression for syndecan-1 in all plasma cells (Fig. 2).

For CD117 immunostaining 6 cases $(60 \%)$ showed grade 1 according to scoring system for immunohistochemical expression in few plasma cells (Fig. 3), while 4 cases (40\%) were grade 0 according Scoring system for immunohistochemical expression for CD117 (Fig. 4).

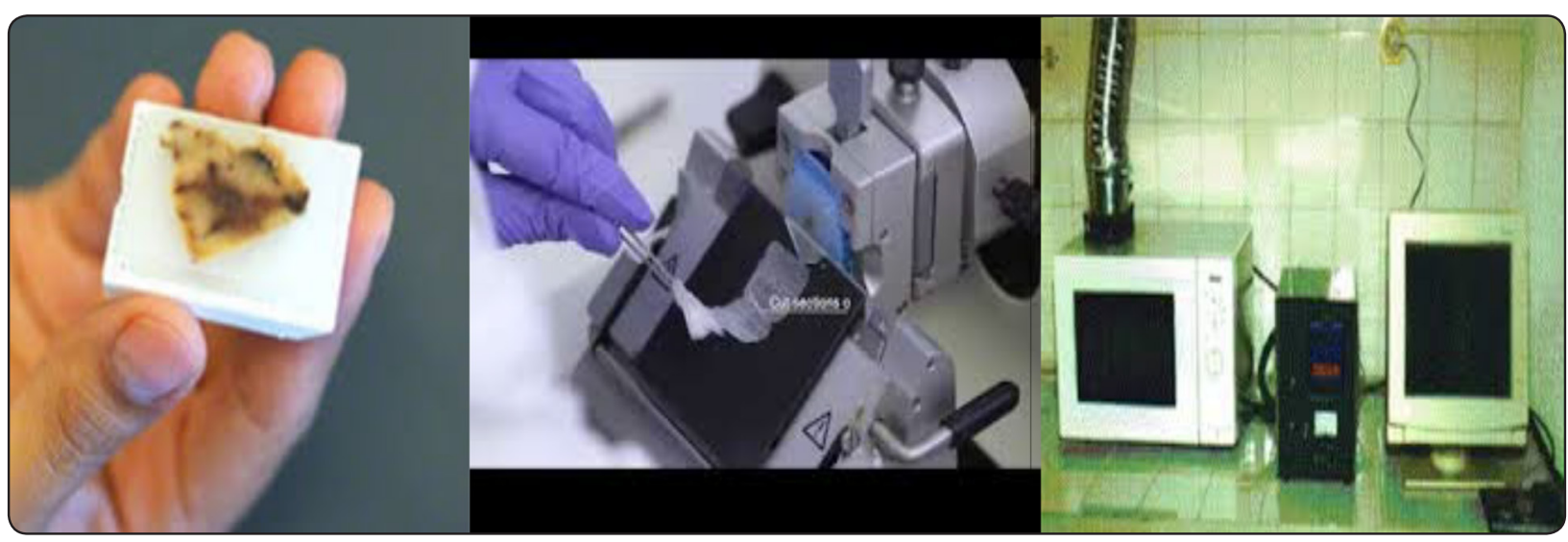

Fig. (1): Showing steps of sectioning and antigen retrieval by microwave 


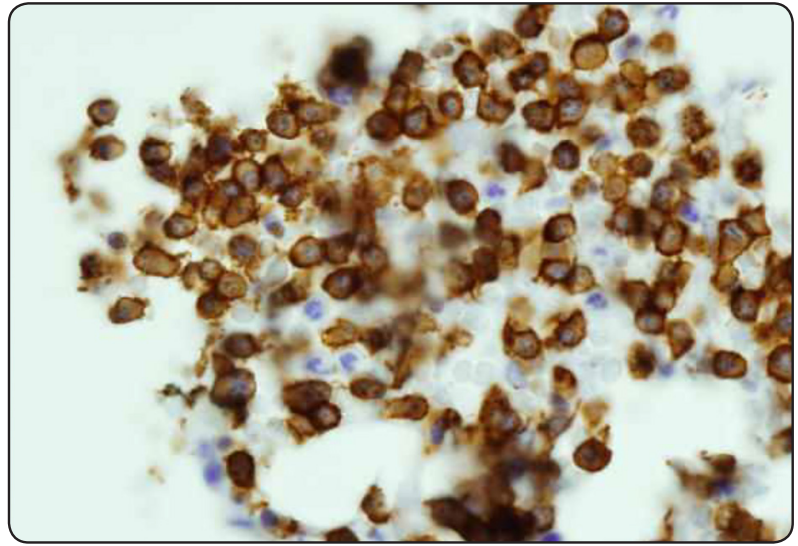

Fig. (2) Photomicrograph showing the expression of syndecan 1 in plasmacytoma showing cytoplasmic and membranous immunostaining in all plasma cells. (X100)

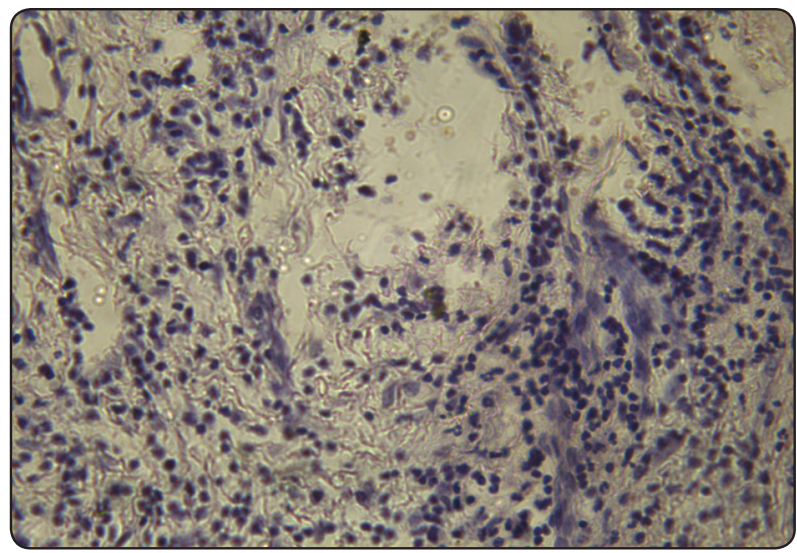

Fig. (3) Photomicrograph showing weak positive cytoplasmic staining in few plasma cells (X40)

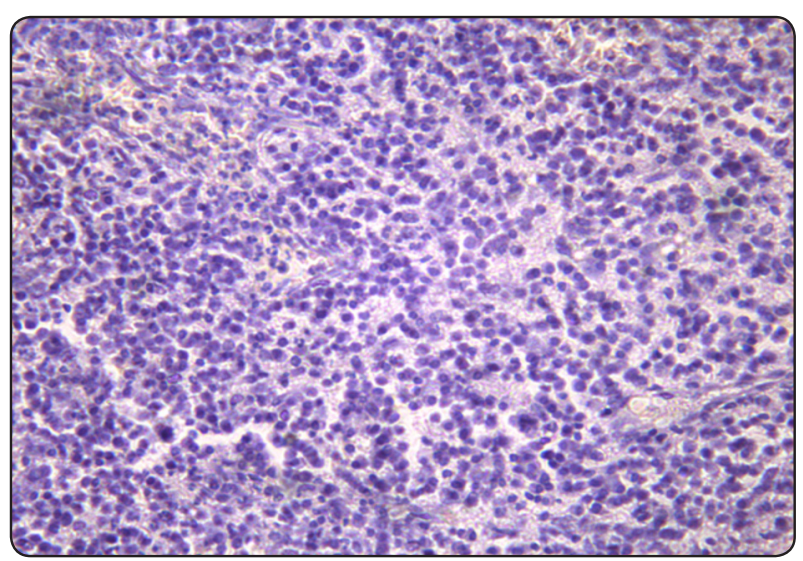

Fig. (4) Photomicrograph showing negative staining for CD117 in plasma cells (X40)
The expression of CD56 in plasmacytoma was similar to the expression of CD117, (60\%) showed grade 1 according to scoring system for immunohistochemical expression in few plasma cells (Fig. 5), while four cases (40\%) were grade 0 according Scoring system for immunohistochemical expression (Fig. 6).

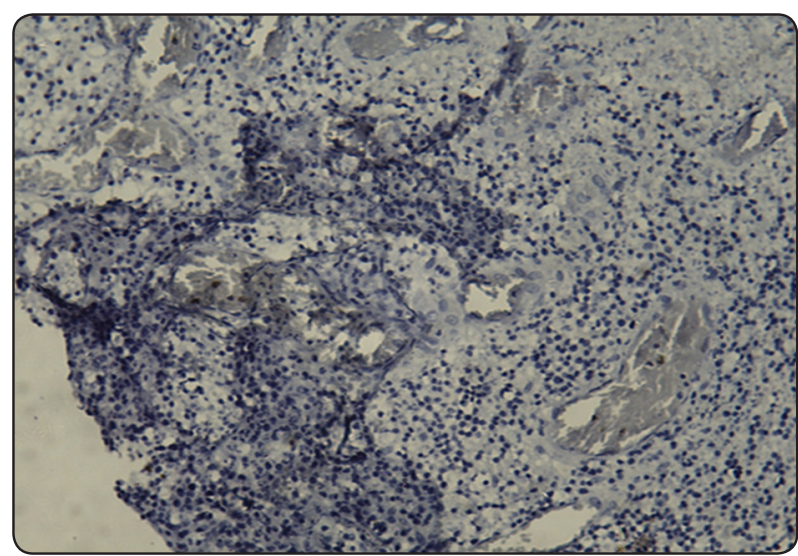

Fig. (5) Photomicrograph showing weak positive cytoplasmic staining for CD56 in few plasma cells (X40)

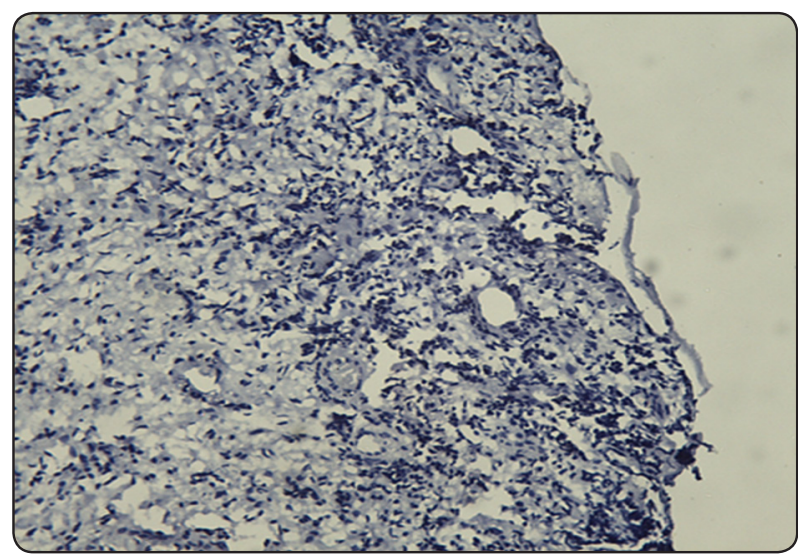

Fig. (6) Photomicrograph showing negative staining for CD56 in plasma cells (X40)

\section{DISCUSSION}

Lesions of MM may be primary or metastatic (21) and the prognosis of solitary plasmacytoma better than the prognosis of MM ${ }^{(22)}$ so early diagnosis is a very important step to differentiate between them ${ }^{(21)}$. 
The prognosis of solitary plasmacytoma become worse if recurrence occurred toward systemic $\mathrm{MM}^{(23)}$. There are many factors that control the evolution to MM, which are size ${ }^{(21)}$, site in bone, old age above 60 years, and the presence of paraprotein at the time of diagnosis ${ }^{(24,25)}$.

As Regard the size, cases of SBP, with size more than 4-5 $\mathrm{cm}$ have a higher tendency to become $\mathrm{MM}^{(26)}$.

The treatment of choice for SBP is the radiotherapy. The combination of surgical removal and chemotherapy is recommended for specific cases. Follow-up is essential for the patients for at least 3 years after diagnosis due to the possibility of developing MM ${ }^{(21)}$. The first step for malignant transformation is residing of plasma cells in the bone marrow. And then extramedullary manifestations such as development of plasmacytomas occur ${ }^{(27)}$.

Most common sites for SBP is the head and neck region about $90 \%$, especially in the upper respiratory tract which include the nasal cavity, sinuses, oropharynx, salivary glands and larynx ${ }^{(28-30)}$.

The objective of the present study was the prognostic evaluation of CD117 and CD 56 in plasmacytoma.

In this study all cases showed strong positive immunoreaction for syndecan 1 . This was in accordance with Cambon $\mathbf{K}$ et $\mathbf{a l}^{(\mathbf{1 1})}$ that mentioned Syndecan-1 acted as a sensitive marker for plasmacytomas, independent of cytologic differentiation.

As well Seidel $\mathbf{C}$ et al ${ }^{(31)}$ and Lovell $\mathbf{R}$ et al ${ }^{(32)}$ said that in patients with MM, soluble syndecan-1 is found in plasma cells and is a prognostic factor ${ }^{(24,25)}$. Also, Kraj M et al ${ }^{(33)}$ found in their study that $100 \%$ patients of plasma cell leukemia (PCL) express syndecan 1 .

For expression of CD117 and CD56 in our study $(60 \%)$ of cases showed weak positive cytoplasmic staining in few plasma cells and (40\%) were negative.
Kraj M et al ${ }^{(33)}$ found that only seven cases out of twenty-three (30\%) evaluated plasma cell leukemia (PCL). Plasma cells showed CD117 expression, while in 16 cases (70\%) patients did not. As CD117 may not be detected on normal plasma cells, but can be detected on some neoplastic plasma cells.

CD117 was associated with good prognosis because the expression of CD117 act as an anchor molecule that result in decrease in spreading of plasma cells ${ }^{(34,35)}$.

While the expression of CD56 in PCL patients does not show uniform pattern, positive reaction of CD56 was found in primary PCL as compared to secondary PCL.

Also, Pellat-Deceunynck et al. ${ }^{(34)}$ reported lack of CD56 expression on malignant plasma cells in $81 \%$ patients with primary or secondary PCL.

Lack of CD56 expression may be considered to be a sign of disease progression. because CD56 mediate homing of myeloma cells. Lack of CD56 expression means weak interactions between myeloma cell-to-cell and myeloma cell-to-bone, also, CD56-cells secret high levels of MMP-9, which leads to degradation of basal membrane and malignant cell spreading ${ }^{(33,36)}$.

The previous studies showed that Lack of CD56 expression in MM cases were associated with higher levels of serum $\beta 2$-microglobulin, lower number of platelets, higher incidence of anemia and renal failure ${ }^{(37)}$.

The mechanism was not clearly understood. As a $140 \mathrm{kDa}$ isoform of neural cell adhesion molecule (NCAM), expressed on the surface of NK cells, CD4+/CD8+ T cells and tumor cells. Regarding plasma cells, it is an example of foreign antigen and its presence gives a mark of malignancy on plasma cell ${ }^{(38)}$.

Ying Pan et al. ${ }^{(39)}$ found $74 \%$ cases of MM were positive for CD56, 32\% for CD117. And also 
found that there was non-significant differences between CD56 positive and CD56 negative cases about monoclonal protein isotype, albumin, $\beta 2$ microglobulin, stage of disease and renal failure. However there was 4 patients lack CD56 expression on their malignant plasma cell as the disease progress. While about CD117 they found lack of expression of CD117 was strongly present with high levels of serum creatinine.

It is stated that patients who express CD56 or CD117 at the new cases were associated with good prognosis in patients of MM, and their absence on malignant plasma cells was associated with poor prognosis in patients of $\mathrm{MM}^{(39)}$.

\section{CONCLUSION}

Although occurrence of SBP is a rare lesion in the bones of the face, the histopathological biopsy for early diagnosis and prognosis evaluations are a must. Also, periodic follow-up of the patient is necessary for at least 3 years after initial diagnosis due to the ability of solitary plasmacytoma to give MM. Syndecan-1can be used as diagnostic marker for plasma cells, while CD56 and CD117 expressed only in neoplastic plasma cells and their expression indicate good prognosis.

\section{RECOMMENDATION}

We recommended performing this study on wider group to confirm the results as the rare occurrence of this neoplasm.

\section{REFERENCES}

1. Ahnach M, Marouan S, Rachid M, Madani A, Quessar A, Benchekroun S, et al. Extramedullary plasocytoma relapsing at different sites: an unusual presentation. Pan Afr Med J. 2013;14:34-7.

2. Ozdemir R, Kayiran O, Oruc M, Karaaslan O, Koc ser U, OgunD. Plasmacytoma of the hard palate. J Craniofac Surg.2005; 16:164-9

3. Ocqueteau, M. ,A. Orfao, R. Garcia-Sanz, J.Almeida , M. Gonzalez, and J. F. San Miguel . Expression of the CD117 antigen (c-Kit) on normal and myelomatous plasma cells Br J Haematol 1996. 95:489-493.

4. Lux, M. L. , B. P. Rubin , and T. L. Biase . et al. KIT extracellular and kinase domain mutations in gastrointestinal stromal tumors. Am J Pathol 2000. 156:791-795.

5. Longley, B. J. , M. J. Reguere , and M. Yongsheng . Classes of c-Kit activating mutations: proposed mechanisms of action and implications for disease classification and therapy. Leuk Res 2001. 25:571-576.

6. Hallek M, Bergsagel PL, Anderson KC. Multiple myeloma: increasing evidence for a multistep transformation process. Blood. 1998;91(1):3-21.

7. S A Pileri, S Ascani, L Leoncini, E Sabattini, P L Zinzani, P P Piccaluga, A Pileri, Jr, M Giunti, B Falini, G B Bolis, and H Stein; Hodgkin's lymphoma: the pathologist's viewpoint, J Clin Pathol. 2002 Mar; 55(3): 162-176.

8. Lux, M. L. , B. P. Rubin , and T. L. Biase . et al. KIT extracellular and kinase domain mutations in gastrointestinal stromal tumors. Am J Pathol 2000. 156:791-795.

9. Ocqueteau, M. , A. Orfao , and J. Almeida . et al. Immunophenotypic characteristics of plasma cells from monoclonal gammopathy of undetermined significance patients: implications for the differential diagnosis between MGUS and multiple myeloma. Am J Pathol 1998. 152:1655-1665.

10. Lemoli, R. M. and A. Fortuna . C-kit ligand (SCL) in human multiple myeloma cells. Leuk Lymphoma 1996. 20:457-464.

11. Cambon K, Venero C, Berezin V, Bock E, Sandi C. Posttraining administration of a synthetic peptide ligand of the neural cell adhesion molecule, $\mathrm{C} 3 \mathrm{~d}$, attenuates long-term expression of contextual fear conditioning. Neuroscience. 2003;122:183-191.

12. Ditlevsen DK, Kohler LB, Pedersen MV, Risell M, Kolkova K, Meyer M, Berezin V, Bock E. The role of phosphatidylinositol 3-kinase in neural cell adhesion molecule-mediated neuronal differentiation and survival. J Neurochem 2003;84:546-556.

13. Brümmendorf T, Lemmon V. Immunoglobulin superfamily receptors: cis-interactions, intracellular adapters and alternative splicing regulate adhesion. Curr Opin Cell Biol. 2001;13:611-618

14. Walmod PS, Kolkova K, Berezin V, Bock E. Zippers make signals: NCAM-mediated molecular interactions and signal transduction. Neurochem Res. 2004;29:2015-2035. 
15. Walsh FS, Putt W, Dickson JG, Quinn CA, Cox RD, Webb M, Spurr N, Goodfellow PN. Human N-CAM gene: mapping to chromosome 11 by analysis of somatic cell hybrids with mouse and human cDNA probes. Brain Res. 1986;387:197-200.

16. Nguyen C, Mattei MG, Mattei JF, Santoni MJ, Goridis C, Jordan BR. Localization of the human NCAM gene to band q23 of chromosome 11: the third gene coding for a cell interaction molecule mapped to the distal portion of the long arm of chromosome 11. J Cell Biol. 1986;102:711-715.

17. Ditlevsen DK, Kohler LB, Pedersen MV, Risell M, Kolkova K, Meyer M, Berezin V, Bock E. The role of phosphatidylinositol 3-kinase in neural cell adhesion molecule-mediated neuronal differentiation and survival. J Neurochem. 2003;84:546-556.

18. Ely, S.A. and Knowles, D.M. Expression of CD56/neural cell adhesion molecule correlates with the presence of lytic bone lesions in multiple myeloma and distinguishes myeloma from monoclonal gammopathy of undetermined significance and lymphomas with plasmacytoid differentiation. Am. J. Pathol. 2002; 160: 1293-1299.

19. Pellat-Deceunynck, C., Barille, S., Jego, G., Puthier, D., Robillard, N., Pineau, D. et al. The absence of CD56 (NCAM) on malignant plasma cells is a hallmark of plasma cell leukemia and of a special subset of multiple myeloma. Leukemia. 1998; 12: 1977-1982.

20. Anthony E Rizzardi, Arthur T Johnson, Rachel Isaksson Vogel, Stefan E Pambuccian, Jonathan Henriksen, Amy PN Skubitz, Gregory J Metzger and Stephen C Schmech; Quantitative comparison of immunohistochemical staining measured by digital image analysis versus pathologist visual scoring; Diagnostic Pathology.2012;7:42.

21. Eduardo Madruga Lombardoa, Fábio Luiz Dal Moro Maitob and Cláiton Heitza; Solitary plasmacytoma of the jaws: therapeutical considerations and prognosis based on a case reports systematic survey; Braz J Otorhinolaryngol. 2018;84(6):790---798

22. P. Di Micco, B. Di Micco; UP-DATE ON SOLITARY PLASMACYTOMA AND ITS MAIN

23. DIFFERENCES WITH MULTIPLE MYELOMA; Experimental Oncology 27, 7-12, 2005

24. Longo DL; Plasma cell disorders. In: Principles of Internal Medicine. Harrison 15th Ed; New York: McGraw Hill Inc, 2001; 727-33.
25. Guo S-Q, Zhang L, Wang Y-F, Sun B-C, Zhang L-Y, Zhang $\mathrm{J}$, et al; Prognostic factors associated with solitary plasmacytoma; OncoTargets Ther. 2013;6:1659-66.

26. Reed V, Shah J, Medeiros LJ, Ha CS, Mazloom A, Weber DM, et al; Solitary plasmacytomas: outcome and prognostic factors afterdefinitive radiation therapy; Cancer. 2011;117:4468-74.

27. Tsang RW, Gospodarowicz MK, Pintilie M, Bezjak A, Wells W,Hodgson DC, et a; Solitary plasmacytoma treated with radio-therapy: impact of tumor size on outcome; Int J Radiat OncolBiol Phys. 2001;50:113---20.

28. Chen HF, Wu TQ, Li ZY, Shen HS, Tang JQ, Fu WJ, et al. Extramedullary plasmacytoma in the presence of multiple myeloma: clinical correlates and prognostic relevance. Onco Targets Ther. 2012;5:329-34.

29. Dimopoulos, M.A., Kiamouris, C. \& Moulopoulos, L.A; Solitary plasmacytoma of bone and extramedullary plasmacytoma. Hematology-Oncology Clinics of North America;1999 13, 1249-1257.

30. Brinch, L., Hannisdal, E., Foss Abrahamsen, A., Kvaloy, S. \& Langholm, R. Extramedullary plasmacytomas and solitary plasma cell tumours of bone; European Journal of Haematology; 1990 44,131-134.

31. Seidel C, Sundan A, Hjorth M, Turesson I, Dahl IM, Abildgaard N, al. Serum syndecan-1: a new independent prognostic marker in multiple myeloma. Blood 2000;95:388-92.

32. Lovell R, Dunn JA, Begum G, Barth NJ, Plant T, Moss $\mathrm{PA}$, et al. Soluble syndecan-1 level at diagnosis is an independent prognostic factor in multiple myeloma and the extent of fall from diagnosis to plateau predicts for overall survival. On behalf of the Working Party on Leukaemia in Adults (part of the National Cancer Research Institut Haematological Oncology Clinical Studies Group). Br J Haematol 2005; 130:542-8.

33. Kraj, M., Kopeć-Szlęzak, J., Pogłód, R., and Kruk, B. Flow cytometric immunophenotypic characteristics of 36 cases of plasma cell leukemia. Leuk. Res. 2011; 35: 169-176.

34. Pellat-Deceunynck, C., Barille, S., Jego, G., Puthier, D., Robillard, N., Pineau, D. et al. The absence of CD56 (NCAM) on malignant plasma cells is a hallmark of plasma cell leukemia and of a special subset of multiple myeloma. Leukemia. 1998; 12: 1977-1982 
35. Ying Pan, Huiping Wang, Qianshan Tao, Cui Zhang, Dongdong Yang, Hui Qin, Shudao Xiong, Lili Tao, Fan Wu, Jiakui Zhang, Zhimin Zhai. Absence of both CD56 and CD117 expression on malignant plasma cells is related with a poor prognosis in patients with newly diagnosed multiple myeloma. Leukemia research. 2016; 40: 77-82

36. Bataille, R., Jégo, G., Robilard, N., Barille-Nion, S., Harousseau, J.L., Moreau, P. et al. The phenotype of normal, reactive and malignant plasma cells:Identification of many and multiple myelomas and of new targets for myeloma therapy. Haematol. Hematol. J. 2006; 91: 1234-1240

37. Sahara, N., Takeshita, A., Shigeno, K., Fujisawa, S., Takeshita, K., Naito, K. et al. Clinicopathological and prognostic characteristics of CD56-negative multiple myeloma. Br. J. Haematol. 2002; 117: 882-885

38. Van Riet, I., De Waele, M., Remels, L., Lacor, P., Schots, R., and Van Camp, B. Expression of cytoadhesion molecules (CD56, CD54CD18 and CD29) by myeloma plasma cells. Br. J. Haematol. 1991; 79: 421-427

39. Ying Pan, Huiping Wang, Qianshan Tao, Cui Zhang, Dongdong Yang, Hui Qin, Shudao Xiong, Lili Tao, Fan Wu, Jiakui Zhang, Zhimin Zhai. Absence of both CD56 and CD117 expression on malignant plasma cells is related with a poor prognosis in patients with newly diagnosed multiple myeloma. Leukemia research. 2016; 40: 77-82 